\title{
EXPERIMENTAL MEASUREMENTS OF FAR FIELD COUGH AIRFLOWS PRODUCED BY HEALTHY AND INFLUENZA-INFECTED SUBJECTS
}

\author{
N. Dudalski, A. Mohamed, E. Savory \\ Dept. Mechanical. \& Materials Engineering \\ The University of Western Ontario \\ London, Canada
}

\author{
S. Mubareka \\ Dept. Biological Sciences \\ Sunnybrook Research Institute \\ Toronto, Canada
}

\begin{abstract}
An experimental study of cough airflow fields produced by subjects who had influenza-like illness was conducted. Particle image velocimetry (PIV) and hot wire anemometry (HWA) measurements were taken in the far-field downstream of the mouth of a participant. Droplet sampling was performed at two locations within a large cough chamber, and a nasal swab confirmed the presence of an infection. The present work analyzes data from two separate cohorts, and modest differences were observed between coughs from sick and convalescent participants. The results are also compared to data obtained from a large eddy simulation (LES) which seeks to model the transient behaviour of a human cough.
\end{abstract}

Keywords-Cough; Influenza; Cold; Airflow; Particle image velocimetry; Hot wire anemometry; Bioaerosol; Fluid mechanics

\section{INTRODUCTION}

Surprisingly little is known about the mechanism of respiratory virus transmission and the dispersion of viral bioaerosols, even though such information is critical in healthcare settings during viral outbreaks. Presumed safe separation distances are not based on scientific evidence, yet there is a widespread adoption of the " $3 \mathrm{ft} / 1 \mathrm{~m}$ rule" and the "6ft/2m rule" [1]. Several studies have conducted measurements of cough strength at the mouth and in the near-field region $(\mathrm{x}<$ $60 \mathrm{~mm}$ downstream) [2-7], but such small separation distances between people are atypical and so significant experimental data in the far field region $(x \geq 1 \mathrm{~m}$ ), while lacking, could provide useful information in the development of infection prevention measures and protocols.

The current project seeks to (1) Assess the " $3 \mathrm{ft} / 1 \mathrm{~m}$ rule" by analyzing velocity measurements taken $1 \mathrm{~m}$ downstream, (2) Estimate and quantify the exposure to airborne viruses and (3) develop a computational fluid dynamics model of cough flow and particle transport based on Large Eddy Simulation (LES) [8,9]. The objective is to recruit 50 participants, naturally infected with influenza, conduct laboratory experiments when they are presumed ill ("sick trials") and to repeat the experiments later when they have recovered ("convalescent trials"). The project also aims to recruit a reference cohort of up to 50 healthy volunteers. Participant recruitment and data analysis will continue throughout 2018, therefore, the current paper represents a preliminary report.

\section{Methodology}

\section{A. Experimental facility (The FLUGIE cough chamber)}

The Fluids from Undergraduates Influenza Enclosure (FLUGIE) consists of a $1.81 \mathrm{~m}$ x $1.78 \mathrm{~m} \times 1.81 \mathrm{~m}$ rectangular wooden box (Fig. 1). A pear-shaped opening allows the participant to cough into the chamber with their nose and mouth unobstructed, while a padded head rest and chin rest ensure that the position of participant's head remains constant between individual trials. The walls of the chamber are painted black, except for a glass side wall allowing optical access, and a glass insert on the bottom of the chamber, allowing a thin laser sheet $(\mathrm{t}=1 \mathrm{~mm})$ to pass through base. Titanium dioxide $\left(\mathrm{TiO}_{2}\right)$ particles are aerosolized before they fill the chamber for Particle Image Velocimetry (PIV) measurements.

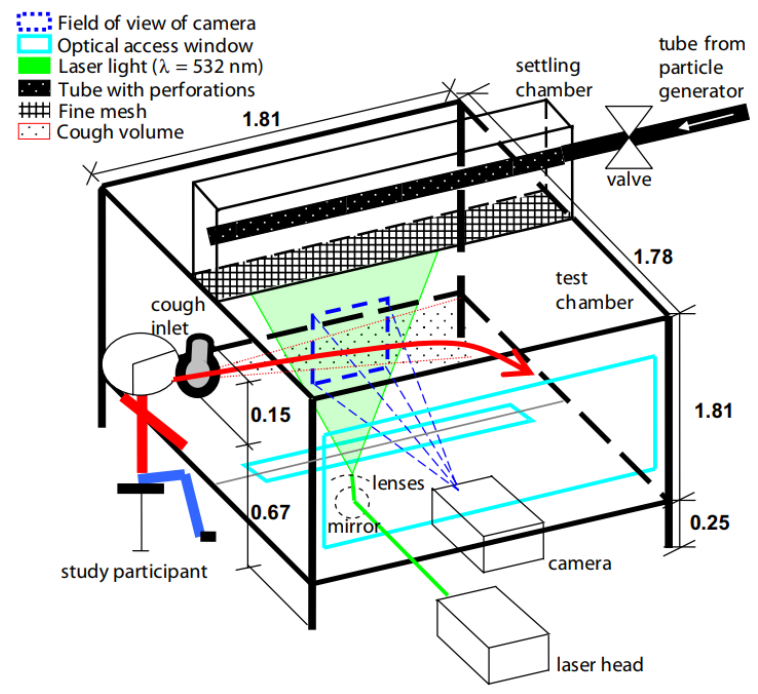

Figure 1: FLUGIE Schematic (All dimensions in m) 


\section{B. Experimental measurements}

After the chamber has been seeded with $\mathrm{TiO}_{2}$ particles, a double-pulsed Nd-Yag laser, operating at $15 \mathrm{~Hz}$, generates a vertical light sheet. The $120 \mathrm{~mJ}$ pulses have a duration of $3-5 \mathrm{~ns}$, and the camera operates with a PIV exposure of 400us. The measured field of view (Fig. 2) is $0.48 \mathrm{~m}$ height $\mathrm{x} 0.89 \mathrm{~m}$ width, and it is centered $1 \mathrm{~m}$ downstream from the participant. Image pairs are captured by a CCD camera (TSI Inc) with a spatial resolution of 6.87 pixels $/ \mathrm{mm}$. Eighty image pairs per cough, are then processed into $102 \times 18$ vector arrays using Insight 4G software. In separate trials, a single hot-wire anemometer (HWA) probe samples velocity readings at $1 \mathrm{kHz}$, at a location $1 \mathrm{~m}$ downstream and $0.170 \mathrm{~m}$ below the inlet centre-line. This probe is calibrated using a separate facility for low air velocity measurements. To assess the presence of viral bioaerosols produced by the violent expiratory events, two low-flow air sampling pumps draw aerosols within the air onto PTFE membrane filters at a constant rate of $4000 \pm 40 \mathrm{~mL} / \mathrm{min}$. The membranes are transferred into individual tubes of UTM viral transport medium. A mid-turbinate swab (MTS) is also collected, to identify the pathogen causing the respiratory infection. All of the sampling tubes are shaken by a vortex shaker before they are stored at $-80^{\circ} \mathrm{C}$. The samples are shipped on dry ice and analyzed by the Dept. of Microbiology at Sunnybrook Health Sciences Centre (Toronto).

\section{Procedure}

Influenza-infected participants are recruited from the clinic at Western Student Health Services after the physicians determine their symptoms to be consistent with an influenza infection. The recruitment procedures are approved by Western's Research Ethics Board (REB approval no. 108945). The inclusion criteria are: age 18-35 and presenting with fever and cough and/or sore throat in the absence of another known cause of illness (e.g. allergies), while exclusion criteria include: immunocompromised, underlying cardiopulmonary disease, pregnancy and smoking. The referred participant is asked to cough 3 times, while aerosol sampling occurs and hot-wire measurements are recorded. There is sufficient time between

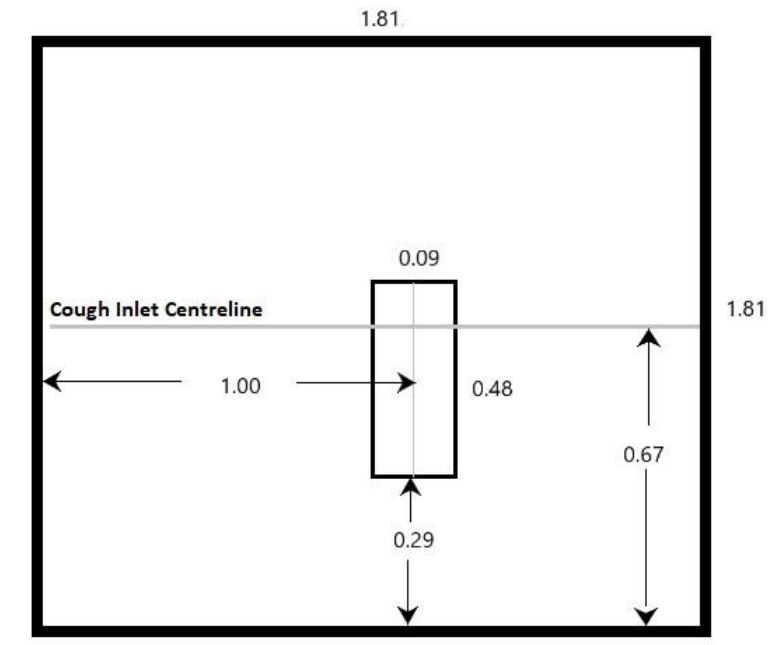

Figure 2: PIV Field of View (All dimensions in m) trials to ensure that residual air motion within the trials is minimal. The hot-wire and sampling cassettes are then removed from the chamber so that the chamber can be seeded for PIV measurements. This is necessary since the seeding particles would damage the hot-wire upon contact. Three additional coughs are then recorded by the PIV system after an even distribution of particles is present within the chamber. Subjects then self-collect a MTS before the experiments are completed. The participant returns after at least 4 weeks, to repeat the hotwire and PIV measurements in their convalescent state. The same procedure is followed for the healthy cohort, but MTS are not collected, and there is no aerosol sampling. Healthy participants also do not return for a second laboratory visit.

\section{RESULTS AND DISCUSSION}

Throughout the 2018 flu season, 7 participants have been recruited to date. From the 7 participants recruited this year, we have collected quality PIV data for 14 coughs. Coughs were excluded from this analysis if the entire width of the expired jet was not contained vertically within the field of view, and if the bulk velocity within the jet was lower than $0.2 \mathrm{~m} / \mathrm{s}$, since in this scenario, it is difficult to distinguish the jet from ambient fluid motion within the chamber.

Each cough was examined, and boundaries that contain the entire jet were specified. Velocity profiles at the midpoint of these boundaries, where the highest velocities are noticed, were extracted at $1 \mathrm{~m}$ downstream. Fig. 3 shows these profiles plotted with respect to time. The velocity obtained from the LES simulation is shown on the same plot for comparison. Boundary conditions for the LES were selected according to conditions of a representative cough based on [10]. A mouth diameter of $3 \mathrm{~cm}$ was specified, with a maximum, transient inlet velocity of $20 \mathrm{~m} / \mathrm{s}$. The average peak velocity for the PIV results is $1.4 \mathrm{~m} / \mathrm{s}$, at $1 \mathrm{~m}$ downstream, which is considerably higher than the $1.1 \mathrm{~m} / \mathrm{s}$ obtained from the LES. The difference may be attributed to the selection of the LES conditions as the average of coughs from 12 female and 13 male participants, whereas the experimental coughs each had different inlet velocities. The presence of

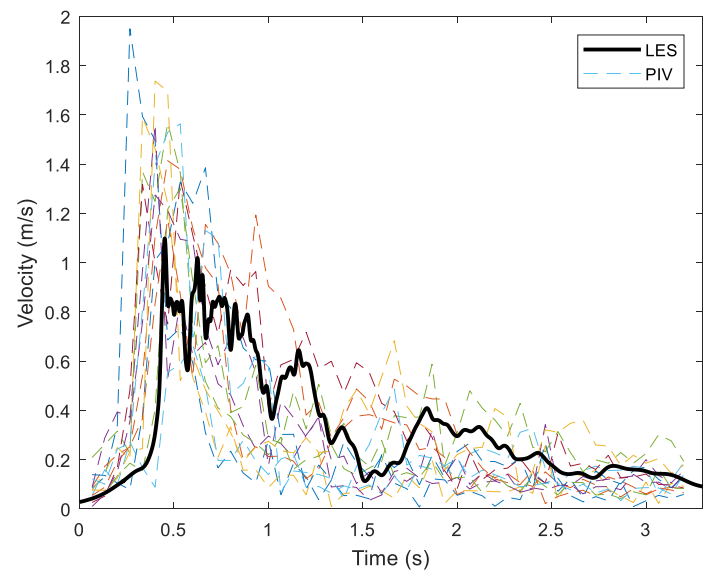

Figure 3: PIV Jet Centre 2D Velocity Magnitudes for Individual Trials 


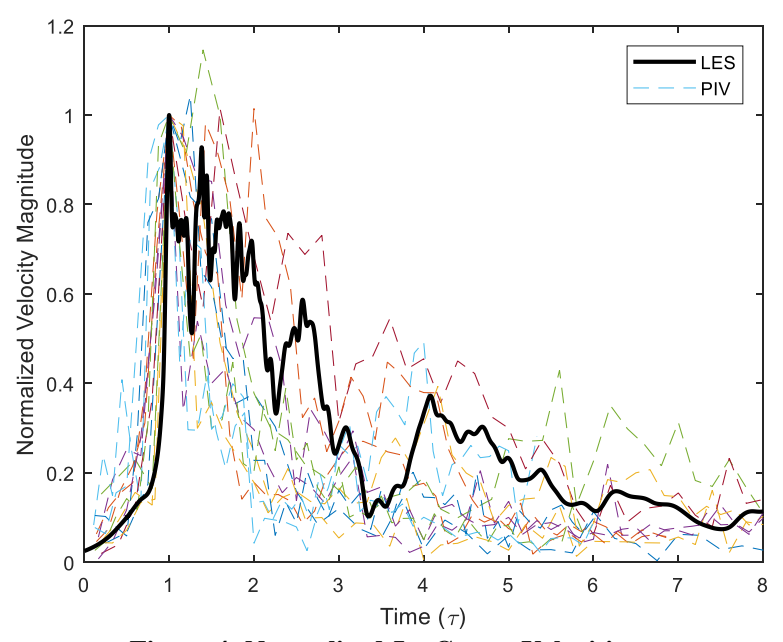

Figure 4: Normalized Jet Centre Velocities

several very strong coughs produced by a couple of participants has influenced the mean, and it is believed that as more participants are recruited, the average maximum velocity at this location will more closely align with the LES. The velocity profiles were normalized by the initial peak $2 \mathrm{D}$ velocity magnitude $\left(\left\langle\mathrm{V}^{\prime}\right\rangle=\langle\mathrm{V}\rangle \mid\langle\mathrm{V}\rangle_{\text {peak }}\right)$, and the time that the peak occurs $\left(\tau=\mathrm{t} / \mathrm{t}_{\text {peak }}\right)$ (Fig. 4). Following normalization, it may be seen that the LES profile more closely resembles those obtained experimentally, but there are data available for many more time steps within the LES. The LES exhibits a secondary peak, which is observed experimentally as well, but this secondary peak is closer in velocity magnitude to the initial peak, and in some trials, it surpasses the velocity of the first peak. The velocity magnitude within the jet was spatially averaged and normalized (Fig. 5). The plot, again, shows a reasonable agreement between the LES and experimental data, although a large variability was observed experimentally.

In order to obtain a better understanding of the spread, and angle of entry of the studied cough jets, U-component velocity measurements were averaged across all $\mathrm{x}$-values, for each $\mathrm{y}$ value $\left(\left\langle U_{\mathrm{x}}{ }^{\prime}\right\rangle\right)$. These profiles were then normalized by the maximum average $U$ velocity and the time that it occurs (Fig. 6). These directionally-averaged profiles were translated so that the jet was centered on the inlet axis. The average distance that the profiles were translated was $0.179 \mathrm{~m}$, indicating that the hot-wire anemometer was appropriately placed to measure velocities at the centre of the jet. An average jet entry angle of 10.2 degrees below the horizontal centreline was calculated from basic trigonometric ratios. Gupta et al. demonstrated that the average cough angle was 40 degrees [10], thus the padded headrest and chin rest successfully reduced the angle, so that the cough enters the chamber roughly horizontally.

The hot-wire data obtained from this cohort requires further analysis before the results can be presented, but hot-wire data was collected from 9 subjects who were recruited during the 2017 flu season. From these, 4 subjects had laboratory confirmed illnesses from the MTS results. Considering the recruitment methods, this is overall a good result. While the other 5 participants exhibited influenza-like symptoms, they might have had bacterial infections like pneumonia or streptococcus, although the presence of these infections was not assessed. The four etiological agents that were found were: coronavirus (CoV) NL63, CoV OC43, Influenza A (H3N2) and respiratory syncytial virus (RSV). Typical velocity time histories are shown for one participant in Fig. 7. The coughs were processed using a moving average filter, with a window size of 299 samples $(0.299 \mathrm{~s})$, so that residual turbulence fluctuations could be separated from the mean cough speed. The peak moving average cough speeds were ranked (Fig. 8), and negligible far field velocities were obtained for about a third of the coughs, in both the sick and convalescent cases. Fig. 9 shows the cumulative average of peak moving average velocity and turbulence intensity of coughs ordered from smallest to largest. The results show that for this cohort, convalescent coughs were slightly stronger but a minimal difference in turbulence intensity was noticed.

\section{CONCLUSION}

At the time of writing 44 participants have been recruited since the study began in 2014. Of these 44, 21 have exhibited influenza-like symptoms and were included in the "sick" cohort. Altogether, HWA data were collected for 48 "sick" coughs, 24 "convalescent" coughs, and 33 "healthy" coughs. PIV data were collected for 29 "sick" coughs, 9 "convalescent" coughs and 15 "healthy" coughs, though much of the data requires further analysis, and were not included in the present paper. MTS and filter cassettes from the most recent cohort of "sick" participants will soon be assessed for viral content. When comparing the preliminary data with the LES model there is reasonable agreement. As more data are collected, it is expected that the agreement will improve, and that it should then be possible to better quantify the differences between coughs from sick and healthy subjects.

\section{ACKNOWLEDGMENTS}

This work is funded by the CIHR/NSERC Collaborative Health Research Program, Canada. Initial funding was provided

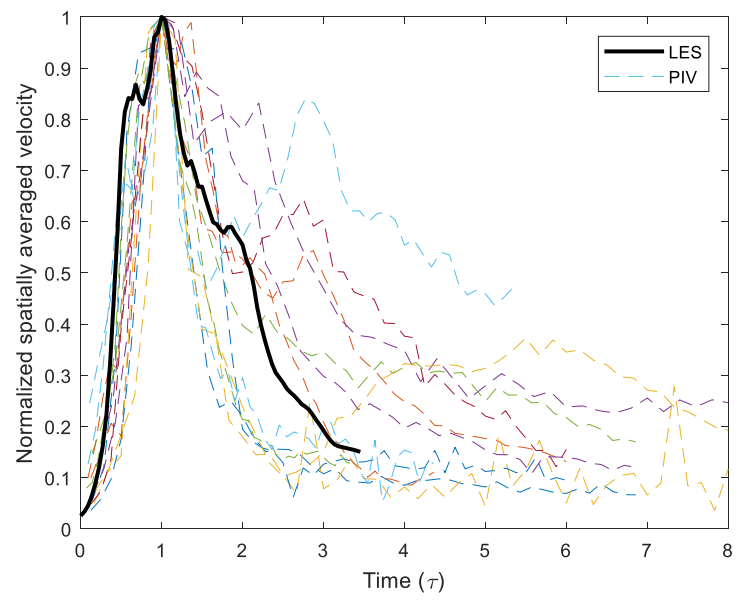

Figure 5: Normalized PIV Spatially Averaged Velocity Magnitudes 
through the Ontario Thoracic Society and the NSERC Engage program. We would also like to express gratitude to the staff at Western Student Health Services for their assistance in participant recruitment, and to Dr. William Lin for his work in conducting previous experiments, and his work in the construction of the experimental facility.

\section{REFERENCES}

[1] Kennamer M: Basic infection control for health care providers. $2^{\text {nd }}$ ed. Albany: Thomson Delmar Learning; 2007.

[2] Zhu S, Kato S, Yang JH: Study on transport characteristics of saliva droplets produced by coughing in a calm indoor environment. Build Environ 2006, 41:1691-1702.

[3] Tang JW, Liebner TJ, Craven BA, Settles GS: A Schlieren optical study of the human cough with and without wearing masks for aerosol infection control. J Royal Society Interface 2009, 6: Suppl 6, S727-S736.

[4] Kwon S-B, Park J, Jang J, Cho Y, Park D-S, Kim C, Bae G-N, Jang A: Study on the initial velocity distribution of exhaled air from coughing and speaking. Chemosphere 2012, 87:1260-1264.
[5] Nishimura H, Sakata S, Kaga A: A new methodology for studying dynamics of aerosol particles in sneeze and cough using a digital highvision, high-speed video system and vector analyses. PLoS One 2013, 8, e80244.

[6] Chao CYH, Wan MP, Morawska L, Johnson GR, Ristovski ZD, Hargreaves M, Mengersen K, Corbett S, Li Y, Xie X, Katoshevski D: Characterization of expiration air jets and droplet size distributions immediately at the mouth opening. J Aerosol Sci 2009, 40:122-133.

[7] VanSciver M, Miller S, Hertzberg J: Particle image velocimetry of human cough. Aero Sci Tech 2011, 45: 415-422.

[8] Savory E, Lin WE, Blackman K, Roberto MC, Cuthbertson LR, Scott JA, Mubareka S: Western Cold and Flu (WeCoF) aerosol study - preliminary results. BMC Res Notes 2014, 7 (1), p. 563.

[9] Bi R, Ali S, Savory E, Zhang C. Computational Fluid Dynamics modeling of human coughs. Proc $25^{\text {th }}$ Annual Conf of CFD Society of Canada, Windsor, Canada, June 2017.

[10] Gupta JK, Lin C-H, Chen Q: Flow dynamics and characterization of a cough. Indoor Air 2009, 19(6):517-525.
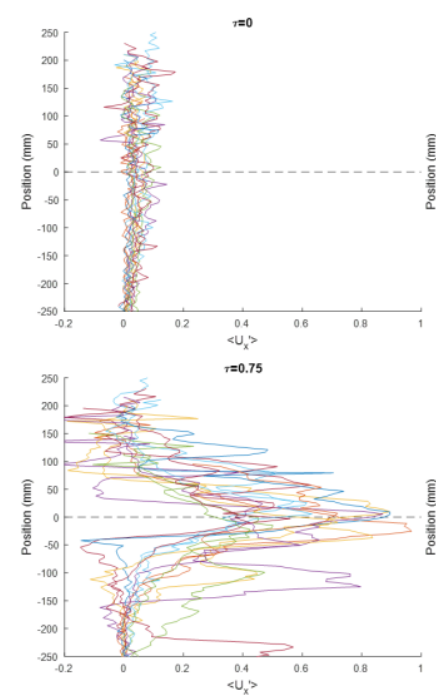

$r=2$
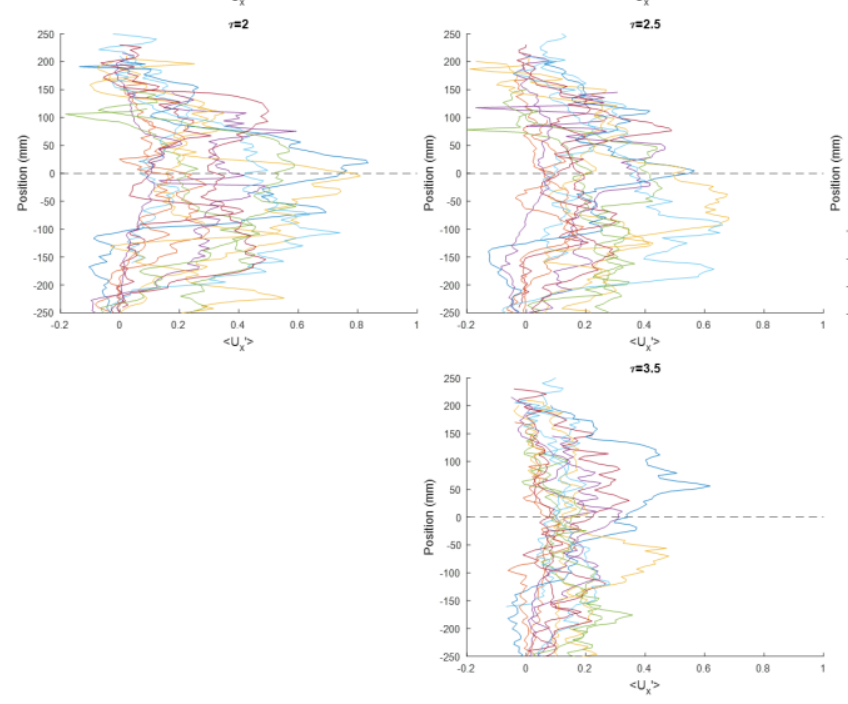

$r=0.25$

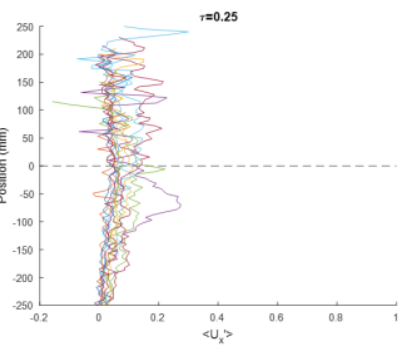

$r=1$

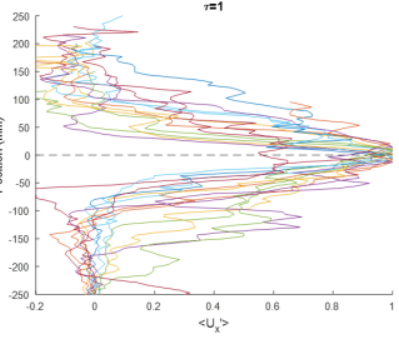

$7=2.5$
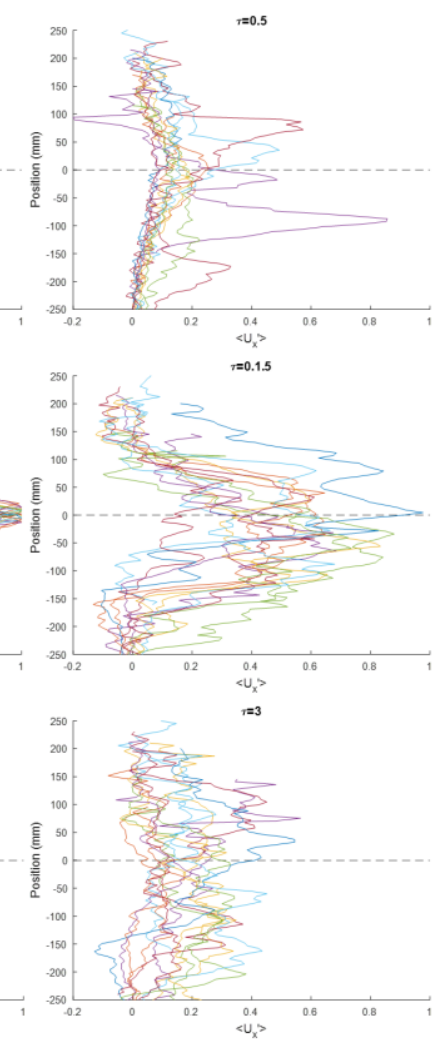

$r=0.1 .5$

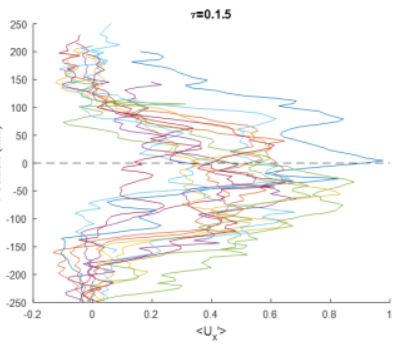

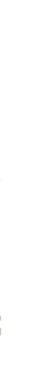

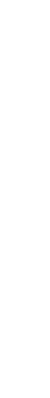

Figure 6: PIV $U$ component velocity averaged across $x$, plotted against $y$ 


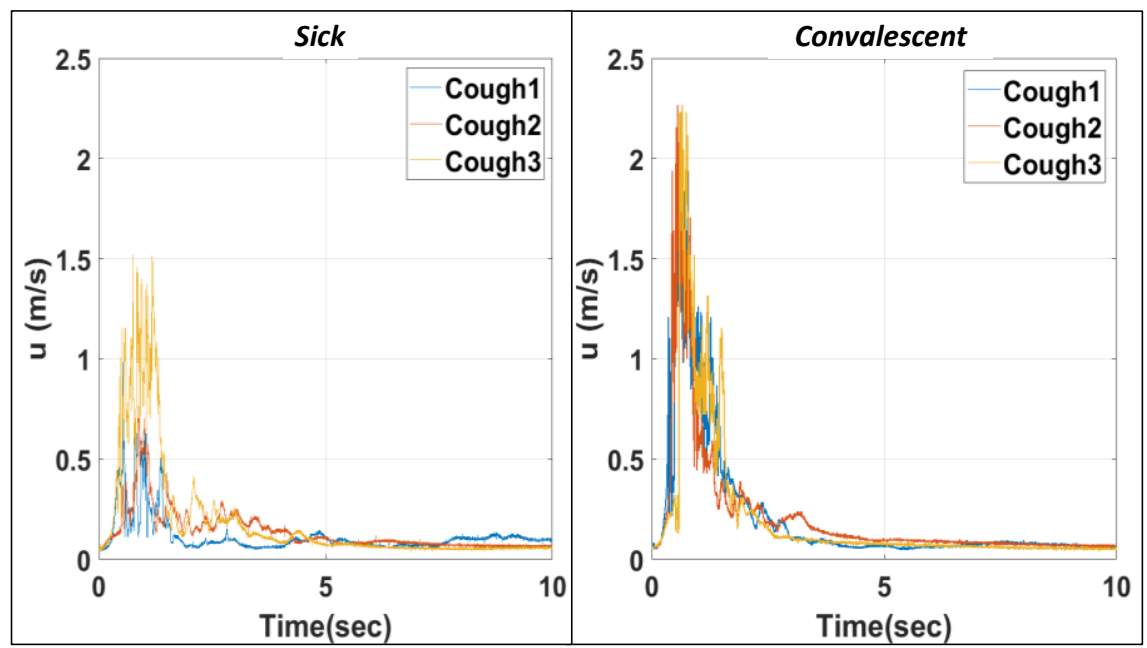

Figure 8: HWA Velocity Profiles

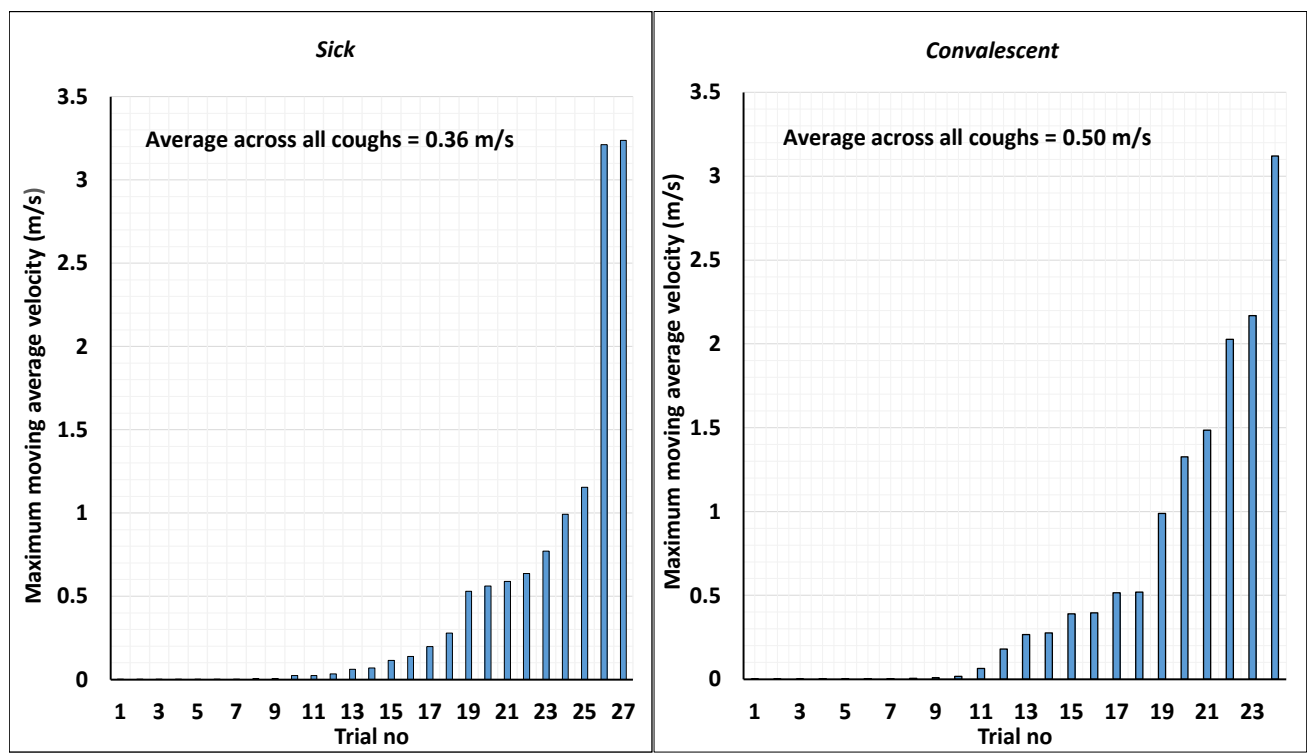

Figure 7: Ranked moving average velocities

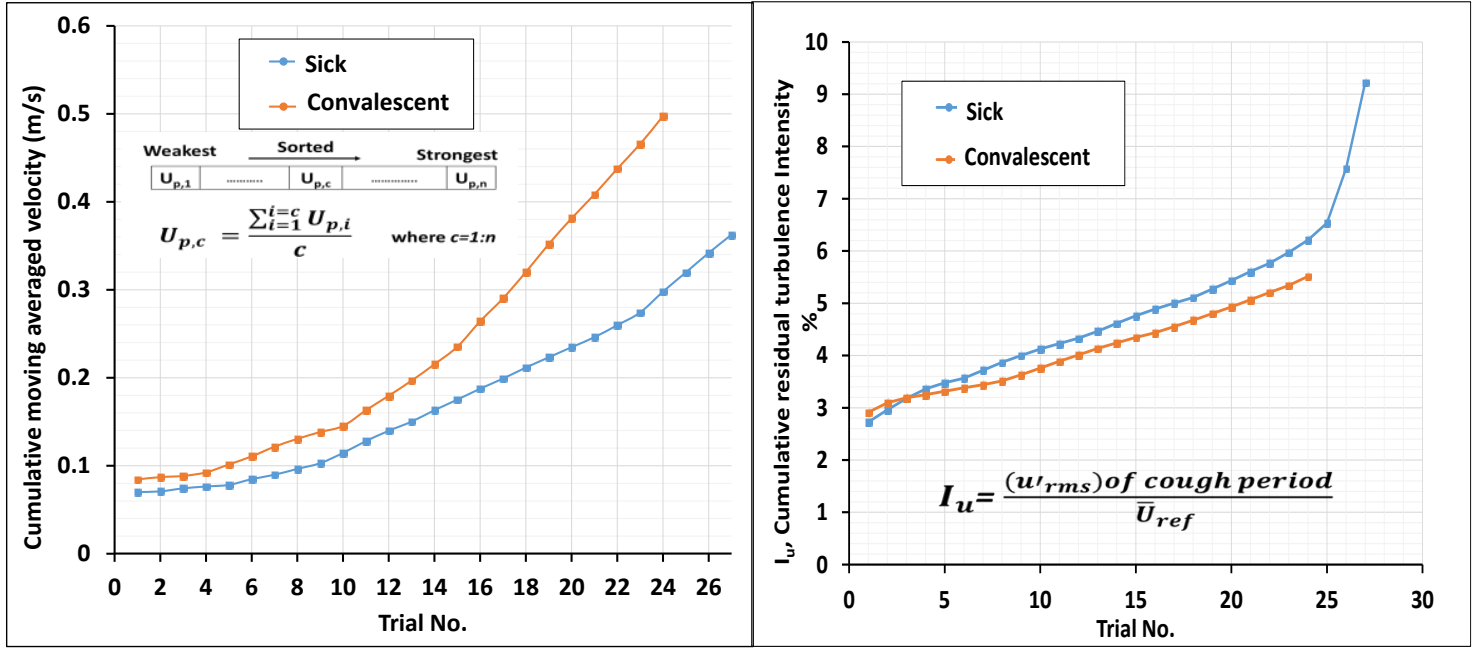

Figure 9: Cumulative averaged velocities and turbulence intensities 